\title{
Static Output Feedback Model Predictive Control for Uncertain Linear Systems
}

\author{
Shuzhan Zhang ${ }^{1}$, Dongya Zhao ${ }^{1}$, Sarah K. Spurgeon ${ }^{2}$ \\ 1. College of New Energy, China University of Petroleum, Qingdao, 266580, P. R. China \\ E-mail: dyzhao@upc.edu.cn \\ 2. Dept. of Electronic and Electrical Engineering, University College London, Torrington Place, London, UK \\ E-mail: s.spurgeon@ucl.ac.uk
}

\begin{abstract}
A static output feedback model predictive control algorithm is proposed for an uncertain linear continuous system. An explicit expression for the static output feedback control law is developed in light of the projection lemma. An infinite time domain optimization problem is transformed into a linear programming problem. The solvability of the optimization problem and the stability are proved to underpin the proposed approach. The effectiveness of the proposed method is validated by using case studies.
\end{abstract}

Key Words: static output feedback, projection lemma, model predictive control, linear matrix inequality

\section{Introduction}

Model predictive control (MPC) is an optimization-based control technique which has attracted much attention because of its ability to handle constraints in an efficient manner [1]. This strategy was originally formulated in the framework of full-state feedback as described in [2] and references therein. In practice, the state may not be fully accessible [4], which can provide a theoretical and/or applications limitation on MPC [3]. When a subset of the system state variables are unknown, a static or dynamic output feedback paradigm is often adopted based on the measured system output information $[5,6]$. The majority of the output based model predictive control approaches adopt dynamic output feedback in the existing literature [7-9].

Compared with dynamic output feedback control, static output feedback control is more straightforward both in terms of design freedom and implementation $[10,11]$. A static output feedback sliding mode control strategy has been developed which uses a particular canonical form for design [12]. Necessary and sufficient conditions for solvability using a static output feedback control have been proposed for linear systems [13], however, an explicit expression to develop a static output feedback control law or optimise a design has not been developed. Sufficient conditions are presented in [14] to develop a stabilising robust static output feedback control. The solution is posed in terms of a set of linear matrix inequalities, but performance optimization is not considered within the problem formulation.

Optimal control is important for the control of industrial processes[15] and MPC has found particular success in process control where it has achieved improved control performance. The purpose of this study is to design a static output feedback model predictive control for a class of continuous linear systems with uncertainties, which explicitly gives an expression for the static output feedback model predictive control law. Compared with the existing methods in [1214], the proposed method not only gives an explicit expression for the control law, but also incorporates an optimiza-

This work is partially supported by the National Natural Science Foundation of China (61973315) and (61473312). tion problem to ensure that the required system performance is maintained in the presence of constraints. The following questions are considered in this paper:

- How to design an explicit expression for a static output feedback model predictive control law according to the necessary and sufficient conditions in [13].

- How to transform an infinite time optimization problem into a problem that can be tractably solved.

After considering these two issues, a step by step control algorithm is presented to facilitate practical design. The effectiveness and performance of the approach developed will be validated using case studies.

The paper is organized as follows. In Section 2, the problem is formulated and related assumptions and lemmas are given. In Section 3, a static output feedback model predictive control algorithm is proposed. The case studies are presented in Section 4 to validate the proposed approach. Finally, some conclusions are drawn in Section 5.

\section{Static output feedback control}

Consider the following continuous-time linear system which is subject to uncertainties:

$$
\begin{aligned}
\dot{x}(t) & =(A+\Delta A) x(t)+(B+\Delta B) u(t) \\
y(t) & =C x(t)
\end{aligned}
$$

where $x(t) \subseteq{ }^{n}$ is the state vector, $u(t) \in \subseteq{ }^{m}$ is the control input and $y(t) \in \subseteq \quad p$ is the output vector. and are polyhedral and polytopic constraint sets, respectively, and $A \in{ }^{n \times n}, B \in{ }^{n \times m}$ and $C \in \quad p \times n$ define the nominal state space description. $\Delta A \in{ }^{n \times n}$ and $\Delta B \in \quad n \times m$ distribute the uncertainty and have the following form:

$$
\left[\begin{array}{cc}
\Delta A & \Delta B
\end{array}\right]=D F(t)\left[\begin{array}{ll}
E_{1} & E_{2}
\end{array}\right]
$$

where $F(t) \in \quad d \times e$ is an unknown bounded function with $F(t)^{T} F(t) \leq I$ and $D \in \quad n \times d, E_{1} \in \quad e \times n$ and $E_{2} \in$ $e \times m$ are known constant matrices. $C$ has full row rank and $B$ has full column rank.

$$
C H=\left[\begin{array}{ll}
I & 0
\end{array}\right]
$$


where $H \in \quad n \times p$ is invertible and $I$ is the unit matrix. For a given nominal triple $(A, B, C)$, necessary and sufficient conditions for the solvability of the constrained Lyapunov problem have been given in [13]. The conditions of the following lemma are required if there is a static output feedback gain $K \in \quad m \times p$, such that:

$$
P(A+B K C)+(A+B K C)^{T} P<0
$$

where $P \in{ }^{n \times n}$ is a symmetric positive definite (SPD) matrix and subject to the equality constraint:

$$
B^{T} P=N C
$$

where $N \in \quad m \times p, K, P$, and $F$ are variables.

Lemma 1 For system (1), the static output feedback gain $K$ exists if and only if: $\operatorname{rank}(C B)=m$ and no invariant zeros of the triple $(A ; B ; C)$ lie in $\mathbb{C}_{+}[13]$.

Remark 1 In [13], although necessary and sufficient conditions are given for the solvability of the static output feedback control law, an explicit expression for the control law has not been developed. In this paper, an explicit expression of the control law is derived from (4) without equality constraints. In order to develop this expression, the projection lemma in [16] will be used in the work which follows.

Based on Lemma 1, a static output feedback controller for the following form can be established:

$$
u(t)=K y(t)
$$

Lemma 2 Suppose that $E$ is a positive definite matrix, the following two expressions are equivalent [16]:

(1) $\Psi+S+S^{T}<0$ is solvable

(2) $\left[\begin{array}{cc}\Psi+E-(W+W)^{T} & S^{T}+W^{T} \\ * & -E\end{array}\right]<0$ is solvable.

Theorem 1 Assume the conditions of Lemma 1 hold for the system (1). If there exist positive definite matrices $M=$ $\left[\begin{array}{cc}M_{11} & 0 \\ M_{21} & M_{22}\end{array}\right]$ and $L=\left[\begin{array}{cc}L_{1} & 0\end{array}\right]$ satisfying:

$$
\begin{gathered}
{\left[\begin{array}{cc}
-G M-(G M)^{T} & \\
* & \\
* & \\
(A T M+B L)^{T}+P^{-1} & (G M)^{T} \\
-P^{-1} & 0 \\
* & -P^{-1}
\end{array}\right]<0}
\end{gathered}
$$

then there is a static output feedback control law $u(t)=$ $L_{1} M_{11}^{-1} y(t)$ that makes the system (1) asymptotically stable.

\section{Proof:}

Applying Lemma 2 to (4) where the state matrices are assumed to include the uncertainty from (1) yields

$$
\begin{aligned}
& {\left[\begin{array}{l}
X-W-W^{T} \\
* \\
{[(A+\Delta A)+(B+\Delta B) K C]^{T} P+W^{T}} \\
-X
\end{array}\right]<0}
\end{aligned}
$$

where $X$ is positive definite matrix. According to the Schur Complement Lemma, (7) is equivalent to:

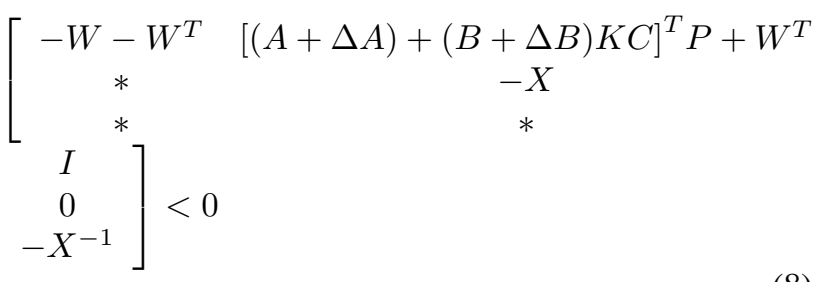

Multiplying both sides of (8) by the diagonal matrix $\operatorname{diag}\left\{I, P^{-1}, I\right\}$ yields:

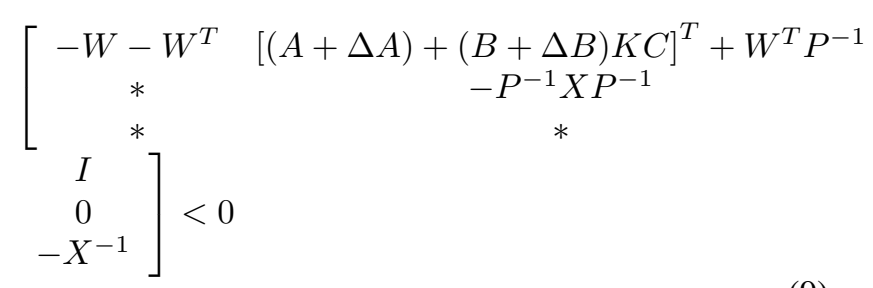

Let $X=P$. Multiplying both sides, in turn, by diagonal matrices $\operatorname{diag}\left\{W^{-T}, I, I\right\}$ and $\operatorname{diag}\left\{W^{-1}, I, I\right\}$ gives

$$
\begin{aligned}
& {\left[\begin{array}{c}
-W^{-1}-W^{-T} \\
* \\
*
\end{array}\right.} \\
& \begin{array}{c}
W^{-T}[A+\Delta A+(B+\Delta B) K C]^{T}+P^{-1} \\
-P^{-1} \\
*
\end{array}
\end{aligned}
$$

Let $W^{-1}=G M$. Then:

$$
\begin{aligned}
& {[A+\Delta A+(B+\Delta B) K C] W^{-1}} \\
& =(A+\Delta A) G M+(B+\Delta B) K C G M \\
& =(A+\Delta A) G M+(B+\Delta B) K\left[\begin{array}{lll}
I & 0
\end{array}\right] M \\
& =(A+\Delta A) G M+(B+\Delta B) K\left[\begin{array}{lll}
M_{11} & 0
\end{array}\right] \\
& =(A+\Delta A) G M+(B+\Delta B)\left[\begin{array}{ll}
L_{1} & 0
\end{array}\right]
\end{aligned}
$$

Hence, $K=L_{1} M_{11}^{-1}$ and $u(t)=L_{1} M_{11}^{-1} y(t)$. Q.E.D.

Remark 2 Inequality (4) prescribes asymptotic stability for the system (1). The static output feedback gain $K=$ $L_{1} M_{11}{ }^{-1}$ can be derived using Lemma 2 from (4). Hence, the control law $u(t)=L_{1} M_{11}{ }^{-1} y(t)$ renders the system (1) asymptotically stable.

\section{Static output feedback model predictive control}

The purpose of this section is to design a static output feedback model predictive control law $u(t)$ for the system (1) so that the state of the system satisfies the following performance index:

$$
\min _{u(t)} J(k)
$$

s.t.

$$
\begin{aligned}
& u_{\text {imin }} \leq u_{i}(k T) \leq u_{\text {imax }}, i=1,2, \cdots, m \\
& y_{\text {jmin }} \leq y_{j}(k T) \leq y_{\text {jmax }}, j=1,2, \cdots, p
\end{aligned}
$$

where

$$
\begin{aligned}
J(k) & =\int_{0}^{+\infty} x^{T}(k T+\tau, k T) R_{1} x(k T+\tau, k T) d \tau \\
& +\int_{0}^{+\infty} u^{T}(k T+\tau, k T) R_{2} u(k T+\tau, k T) d \tau
\end{aligned}
$$


and $R_{1}$ and $R_{2}$ are given real positive definite matrices, $T$ is the sampling period, $\left\{t_{k}\right\}_{k=0,1, \ldots}$ is the sampling time which satisfies $t_{k+1}-t_{k}=T . x(k T)=x(k T, k T)$ is the state at sampling time $k T, x(k T+\tau, k T)$ is the predicted state at $k T+\tau$ at time $k T . u(k T+\tau, k T)$ is the predicted control law for time $k T+\tau$ at time $k T$. It is assumed that there exists a quadratic function $V[x(t)]$ making $J[x(t)] \leq V[x(t)]$. The infinite time domain optimization problem is then transformed into a solvable finite time LMI problem. Consider the following Lyapunov function:

$V(t)=\int_{0}^{+\infty}\left[x^{T}(t) R_{1} x(t)+u^{T}(t) R_{2} u(t)\right] d t+x^{T}(t) P x(t)$

It follows that

$$
\begin{aligned}
\dot{V}(t)=\int_{0}^{+\infty}\left\{x ^ { T } ( t ) \left[R_{1}+(K C)^{T} R_{2}(K C)\right.\right. \\
+P[(A+\Delta A)+(B+\Delta B) K C] \\
\left.\left.\quad+[(A+\Delta A)+(B+\Delta B) K C]^{T} P\right] x(t)\right\} d t
\end{aligned}
$$

if

$$
\begin{aligned}
& R_{1}+(K C)^{T} R_{2}(K C) \\
& +P[A+\Delta A+(B+\Delta B) K C] \\
& +[A+\Delta A+(B+\Delta B) K C]^{T} P<0
\end{aligned}
$$

the system (1) is stable.

Theorem 2 Assume the conditions of Lemma 1 hold for the system (1), If there are positive definite matrices $Y>0$ and $M=\left[\begin{array}{cc}M_{11} & 0 \\ M_{21} & M_{22}\end{array}\right]$ with $L=\left[\begin{array}{cc}L_{1} & 0\end{array}\right]$ satisfying:

$$
\left.\begin{array}{cccc}
-G M-(G M)^{T} & {[(A+\Delta A) G M+(B+\Delta B) L]^{T}+Y} \\
& * & & -Y \\
& * & & * \\
& * & & * \\
& * & & * \\
& * & & 0 \\
(G M)^{T} & (G M)^{T} & L^{T} & (G M+L)^{T} \\
0 & 0 & 0 & 0 \\
-Y & 0 & 0 & 0 \\
* & -R_{1}{ }^{-1} & 0 & 0 \\
* & * & -R_{2}^{-1} & I
\end{array}\right]<0
$$

s.t. (13), (14), then, there exists a static output feedback control law $u(t)=L_{1} M_{11}^{-1} y(t)$ that makes the system (1) asymptotically stable.

Proof:

According to Lemma 2, (17) is equivalent to:

$$
\left[\begin{array}{c}
R_{1}+(K C)^{T} R_{2} K C+X-W-W^{T} \\
* \\
\left.\left[(A+\Delta A)+W^{T}+((B+\Delta B) K C)\right]^{T} P\right]<0 \\
-X
\end{array}\right.
$$

where $X$ is positive definite matrix. According to the Schur
Complement Lemma, (19) is equivalent to:

$$
\left[\begin{array}{ccc}
-W-W^{T} & {[(A+\Delta A)+((B+\Delta B) K C)]^{T} P+W^{T}} \\
* & & -X \\
* & & * \\
* & & * \\
* & & \\
I & I & (K C)^{T} \\
0 & 0 & 0 \\
-X^{-1} & 0 & 0 \\
* & -R_{1}^{-1} & 0 \\
* & * & -R_{2}^{-1}
\end{array}\right]<0
$$

Multipling both sides by the diagonal matrix $\operatorname{diag}\left\{W^{-T}, P^{-1}, I, I, I\right\}$ yields:

$$
\left[\begin{array}{ccc}
-W-W^{T} & \Phi & \\
* & -P^{-1} X & \\
* & * & \\
* & * & \\
* & * & \\
W^{-T} & W^{-T} & W^{-T}(K C)^{T} \\
0 & 0 & 0 \\
-X^{-1} & 0 & 0 \\
* & -R_{1}^{-1} & 0 \\
* & * & -R_{2}^{-1}
\end{array}\right]<0
$$

where $\Phi=W^{-T}[(A+\Delta A)+(B+\Delta B) K C]^{T}+P^{-1}$. Define $X=P, W^{-1}=G M$, then, $K=L_{1} M_{11}{ }^{-1}$ and $u(t)=L_{1} M_{11}^{-1} y(t)$. (18) is satisfied. Q.E.D.

Remark 3 Theorem 2 shows that the problem of static output feedback MPC can be transformed into a "min" optimization problem under a set of linear matrix inequality constraints. Inequality (18) can be solved using the LMI toolbox.

The optimization problem (12) can be transformed into the following LMI:

$$
\min _{Y, M, L, K} V(t), \text { s.t.(13), (14), (18) }
$$

The design procedure can be described in the following step by step algorithm.

Algorithm 1

Step 1 (Initialization): Choose $t_{k}$ and $T$ which satisfy $t_{k}=k T$, set $k=0$.

Step 2 (Updating): At the beginning of the control interval $(k T)$, set the iteration $l=0$ and $K=K^{(l=0)}$, where $l$ is the iteration number.

Step 3 (Iterations): At time step $k T$, solve the LMI (22) to obtain the matrices $Y, L$ and $M$ and feedback gain $K$. Check the convergence for a specified error tolerance $e$ which is defined by the user. If $K^{(l)}$ satisfies:

$$
\left\|K^{(l)}-K^{(l-1)}\right\| \leq e
$$

then, go to step 4. Otherwise continue to iterate, exchange the solution $K$ and set $l=l+1$.

Step 4 (Implementation): Judge whether the constraints are met. If the control gain $K$ cannot meet the constraints 
at time step $k T$, then $K(k T)=K(k T-1)$ until the constraints are satisfied. If $K$ meets the constraints, apply the control $u(k T)=K(k T) y(k T)$ to the system. Go to the control interval $k T=k T+1$, return to step 2 and repeat the procedure.

Remark 4 For a given matrix $C$, the choice of $G$ is not unique. In this paper, define $G=\left[\begin{array}{ll}C^{T}\left(C C^{T}\right)^{-1} & C^{\dagger}\end{array}\right]$ where $C^{\dagger}$ is the orthogonal basis of the zero space of the matrix $C$. G satisfies $C G=\left[\begin{array}{ll}I & 0\end{array}\right]$ and guarantees the feasibility of Step 3 in Algorithm 1.

\section{Case Study}

In this section, the proposed method is verified by case studies. The first case seeks to verify the effectiveness of the proposed approach to stabilise a given system; the second case study verifies the effectiveness of the proposed approach in solving a tracking problem; the third case verifies the effectiveness of the tracking performance in the presence of uncertainty.

\subsection{Stabilization Problem}

Consider the following linear continuous system:

$$
\begin{aligned}
A & =\left[\begin{array}{cc}
1.1 & -0.3 \\
0 & 0.1
\end{array}\right] \\
B & =\left[\begin{array}{c}
-1.5 \\
1
\end{array}\right] \\
C & =\left[\begin{array}{ll}
1 & 0
\end{array}\right] \\
R_{1} & =I \\
R_{2} & =3 I
\end{aligned}
$$

According to the static output feedback model predictive control algorithm in this paper, matrices $G, Y, M$ and $L$ can be obtained by the mincx solver in the LMI Toolbox:

$$
\begin{gathered}
G=\left[\begin{array}{ll}
1 & 0 \\
0 & 1
\end{array}\right] \\
Y=\left[\begin{array}{cc}
1.0395 & -0.0379 \\
-0.0379 & 1.2662
\end{array}\right] \\
M=\left[\begin{array}{cc}
0.4272 & 0 \\
-0.0036 & 0.4236
\end{array}\right] \\
L=\left[\begin{array}{ll}
0.8163 & 0
\end{array}\right]
\end{gathered}
$$

Select the initial state of the system as $x_{0}=\left[\begin{array}{ll}2 & 1\end{array}\right]^{T}$. The states of the closed-loop system are shown in Figure 1, the control input is shown in Figure 2, the output of the system is shown in Figure 3 and the performance is shown in Figure 4. Figure 1 shows that the two states of the system can be rapidly stabilized. From Figure 2 to Figure 4, it can be seen that the system has good performance. This validates the effectiveness of the proposed approach in solving a stabilization problem.

\subsection{Tracking Problem}

Consider the nominal system from (23). Select the reference output trajectory as $0.1 \sin (\pi t / 3)$ and the initial state of the system as $x_{0}=\left[\begin{array}{ll}0.05 & 0\end{array}\right]^{T}$. The output of the

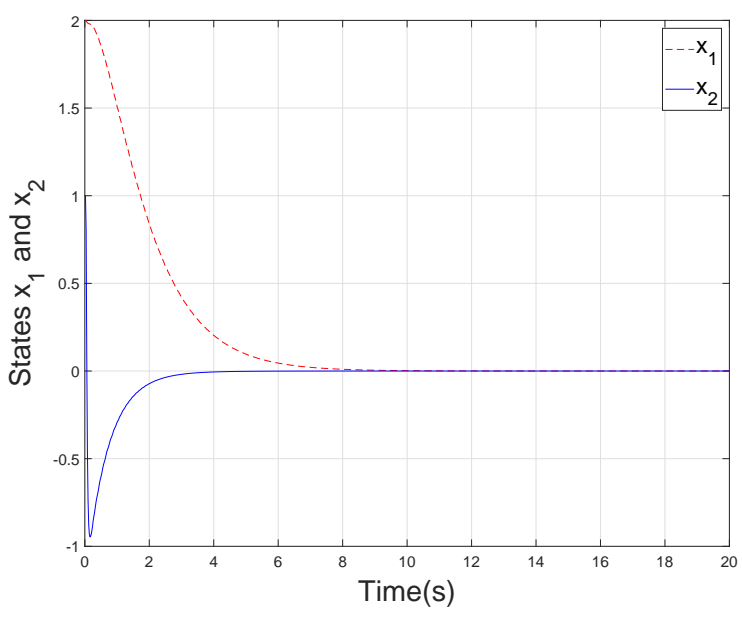

Fig. 1: States of the closed-loop system (Case A).

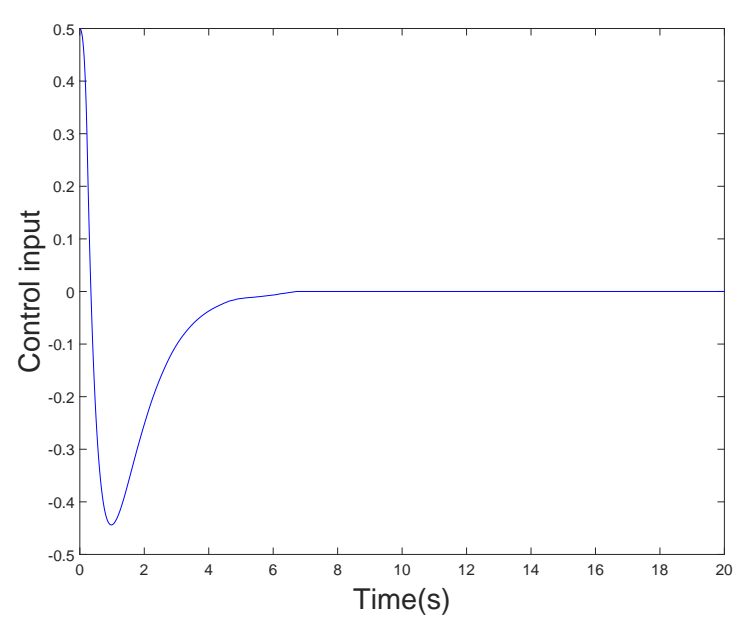

Fig. 2: Control input (Case A).

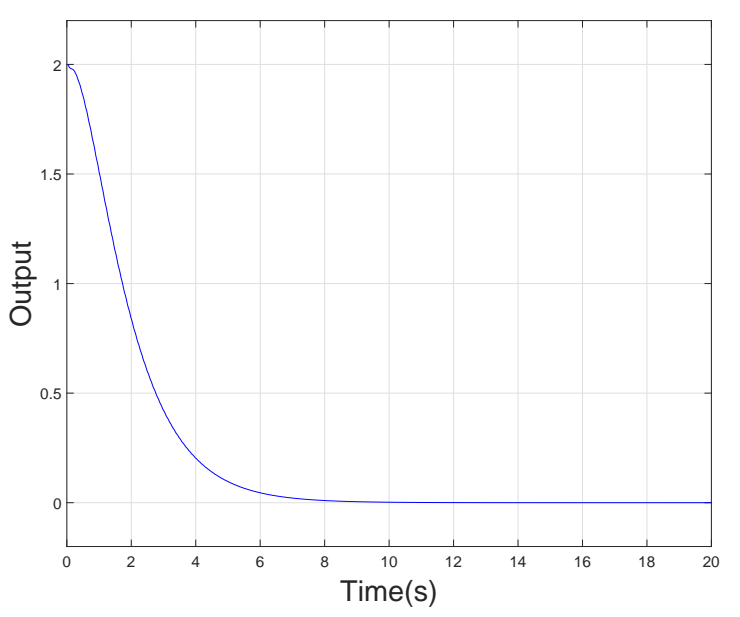

Fig. 3: Output of the closed-loop system (Case A). 


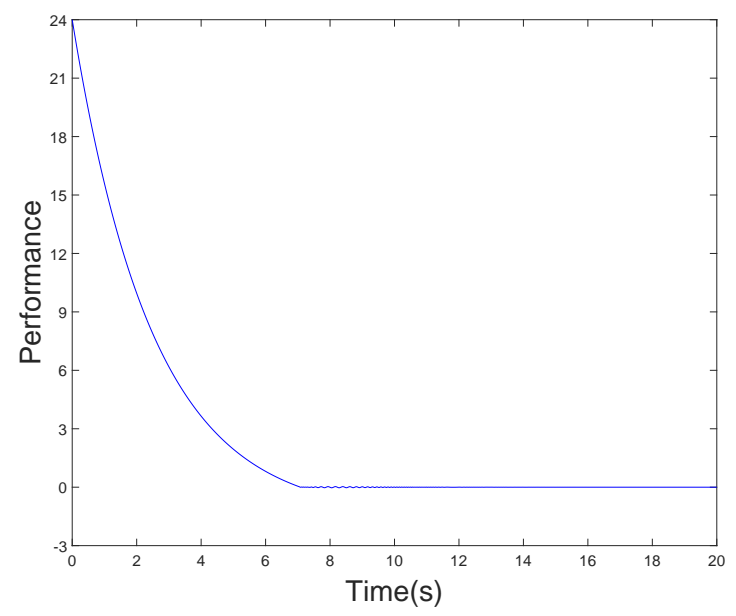

Fig. 4: Performance of the closed-loop system (Case A).

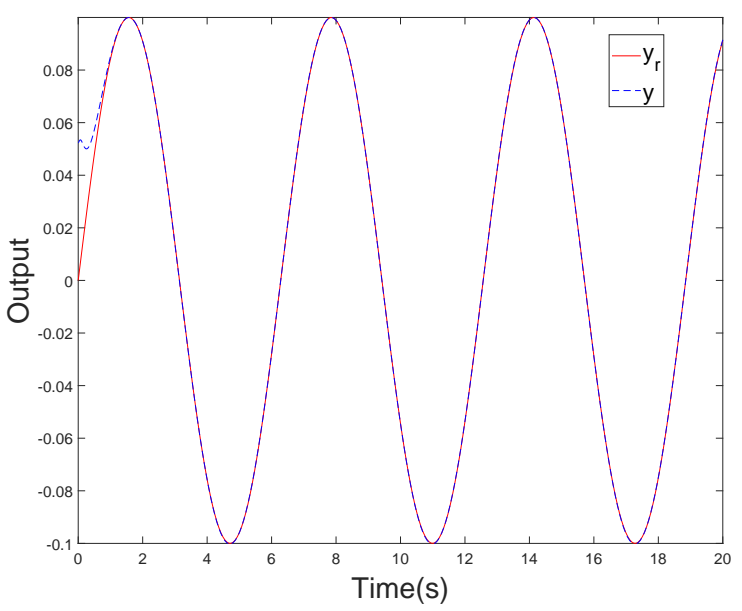

Fig. 5: Output of the closed-loop system (Case B).

closed-loop system is given in Figure 5 and the control input is shown in Figure 6. In Figure 5, the solid line is the reference output and the dashed line is the actual output. It is seen that the output tracks the desired reference rapidly and with high accuracy. The results show that the system exhibits good tracking performance.

\subsection{Tracking Performance in the Presence of Uncer- tainties}

For the system (23), consider now the presence of uncertainties as described in (2) where

$$
\begin{gathered}
E_{1}=\left[\begin{array}{llll}
1 & 0 & 0 & 0 \\
1 & 0 & 0 & 0
\end{array}\right]^{T} \\
E_{2}=\left[\begin{array}{llll}
0 & 1 & 0 & 0
\end{array}\right]^{T} \\
D=\left[\begin{array}{cccc}
0.1 & 0 & 0.1 & 1 \\
0 & 1 & 0 & 0
\end{array}\right] \\
F(t)=\operatorname{diag}\left\{f_{1}, f_{2}, f_{3}, f_{4}\right\}
\end{gathered}
$$

where $\left|f_{1}\right| \leq 0.1,\left|f_{2}\right| \leq 0.1,\left|f_{3}\right| \leq 0.1,\left|f_{4}\right| \leq 0.1$. Select the reference output trajectory as $\cos (\pi t)$ and the initial

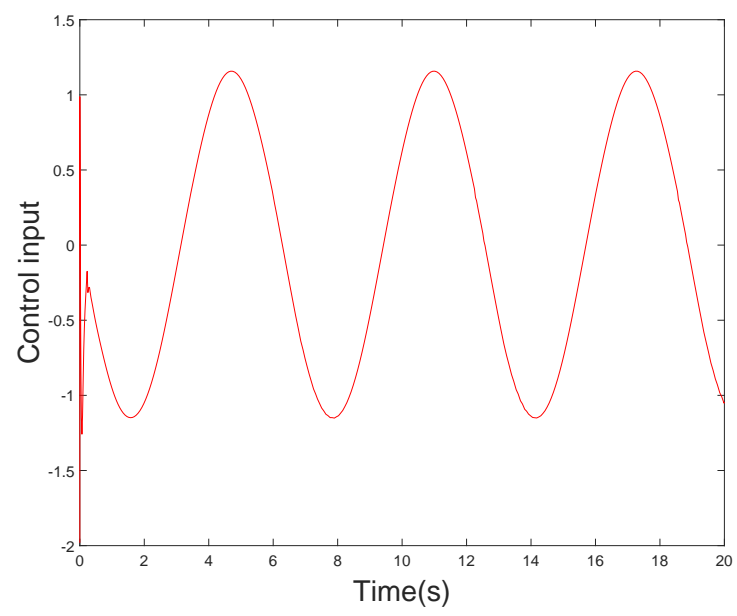

Fig. 6: Control input (Case B).

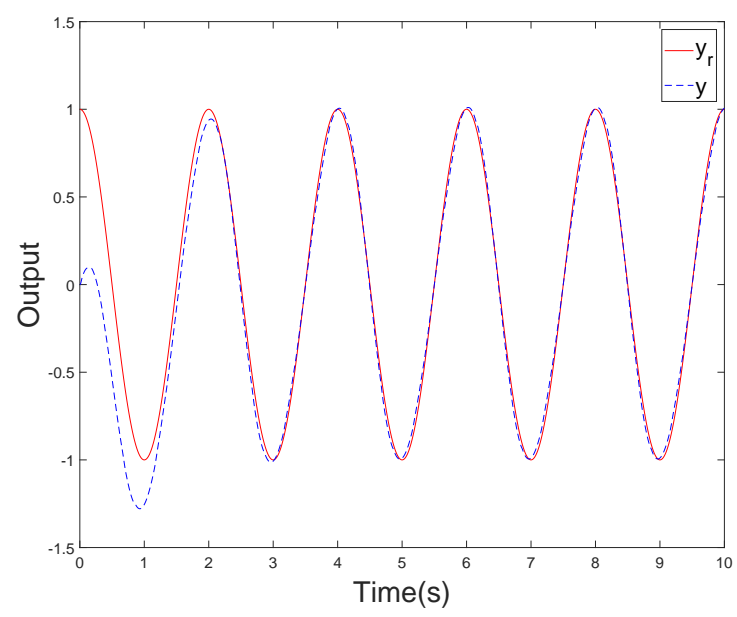

Fig. 7: Output of the closed-loop system (Case C).

state of the system as $x_{0}=\left[\begin{array}{ll}0 & 0\end{array}\right]^{T}$. The output of the closed-loop system is given in Figure 7, the control input is given in Figure 8 and the tracking error is given in Figure 9. Figure 7 shows that the output tracks the desired reference rapidly. Figure 9 shows that the tracking error is bounded and the method exhibits good robustness in the presence of uncertainty.

\section{Conclusion}

A static output feedback model predictive control algorithm is proposed in this paper for the case when the system states are not measurable. The main contribution of this paper can be summarized as: (1) An explicit expression for the static output feedback control law is obtained using the projection lemma. (2) The resulting infinite time domain optimization problem is transformed into a linear programming problem using LMIs and the expression for the static output feedback predictive control law is given. Case studies show the feasibility of proposed approach. Future work will focus on experimental trials of the proposed approach.

\section{References}

[1] S. Lucia, D. Navarro, O. Lucia, P. Zometa, R. Findeisen, "Optimized fpga implementation of model predictive control for em- 


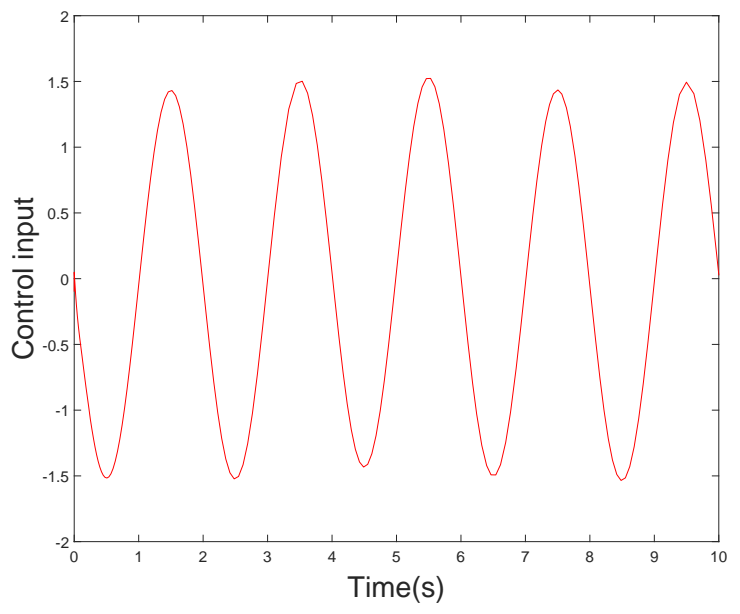

Fig. 8: Control input (Case C).

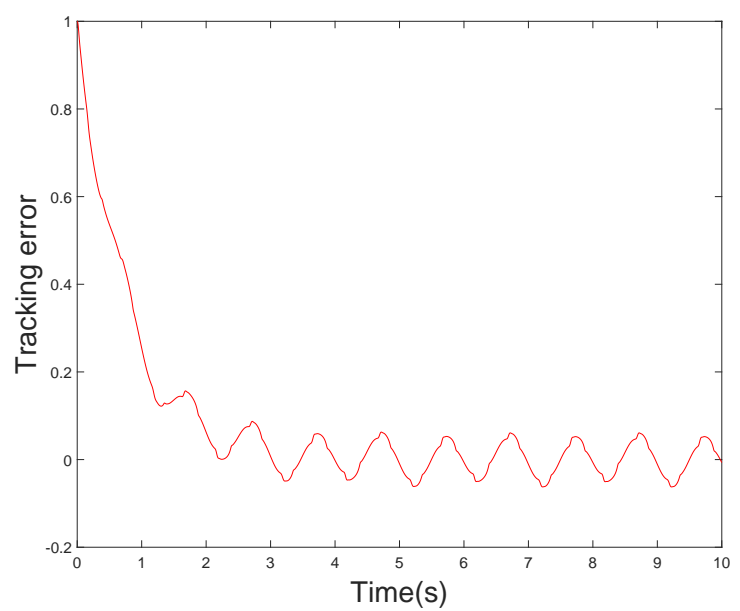

Fig. 9: Tracking error (Case C).

bedded systems using high level synthesis tool," IEEE Transactions on Industrial Informatics, vol. 99, pp. 529-551, 2017.

[2] Martin A. Sehr, Robert R. Bitmead, "Stochastic outputfeedback model predictive control," Automatica, vol. 94, pp. 315-323, 2018.

[3] Mayne,D.Q., "Model predictive control: Recent developments and future promise," Automatica, vol. 50, no. 12, pp. 29672986, 2014.

[4] Z. Wan, M. V. Kothare, "An efficient off-line formulation of robust model predictive control using linear matrix inequalities," Automatica, vol. 39, no. 5, pp. 837-846, 2003.

[5] D. Li, Y. Xi, "The feedback robust mpc for LPV systems with bounded rates of parameter changes," IEEE Transactions on Automatic Control, vol. 55, no. 2, pp. 503-507, 2010.

[6] B. Ding, H. Pan, "Output feedback robust mpc with one free control move for the linear polytopic uncertain system with bounded disturbance," Automatica, vol. 50, no. 11, pp. 29292935, 2018.

[7] Mayne,D.Q.,Rakovic,S.,Findeisen,R.,and Allgower,F, "Robust output feedback model predictive control of constrained linear systems," Automatica, vol. 52, no. 7, pp. 1217-1222, 2006.

[8] Mayne,D.Q, "Robust and stochastic model predictive control: Are we going in the right direction?," Annual Reviews in Control, vol. 41, pp. 184-192, 2016.

[9] Findeisen,R.,Imsland,L.,Allgower,F.,and Foss,B.A, "State and output feedback nonlinear model predictive control: An overview," European Journal of Control, vol. 9, no. 2, pp. 190216, 2003.

[10] K. S. Kim, Y. Park, "Equivalence between two solvability conditions for a static output feedback problem,'JEEE Transactions on Automatic Control, vol. 45, no. 10, pp. 1187-1187, 2000.

[11] X. G. Yan, S. K. Spurgeon, C. Edwards, "Memoryless static output feedback sliding mode control for nonlinear systems with delayed disturbances,'IEEE Transactions on Automatic Control, vol. 59, no. 7, pp. 1906-1912, 2010.

[12] C. Edwards, S. Spurgeon, "Sliding mode stabilization of uncertain systems using only output information,'International Journal of Control, vol. 62, no. 5, pp. 1129-1144, 1995.

[13] C. Edwards, X. G. Yan, S. Spurgeon, "On the solvability of the constrained lyapunov problem,'IEEE Transactions on Automatic Control, vol. 52, no. 10, pp. 1982-1987, 2007.

[14] J. X. Dong, G. H. Yang, "Static output feedback control synthesis for linear systems with time-invariant parametric uncertainties, "IEEE Transactions on Automatic Control, vol. 52, no. 1, pp. 1930-1936, 2007.

[15] Mayne,D.Q.,Rawlings,J.B.,Rao,C.V.,and Scokaert,P.O.M, "Constrained model predictive control: stability and optimality,"Automatica, vol. 36, no. 6, pp. 789-814, 2000.

[16] L. Xie, "Output-feedback h control of systems with parameter uncertainty,'International Journal of Control, vol. 63, no. 4, pp. 741-750, 1996. 